\title{
Low frequency waves in plasmas with spatially varying electron temperature
}

\author{
P. Guio ${ }^{1, *}$, S. Børve ${ }^{2}$, H. L. Pécseli ${ }^{3}$, J. Trulsen ${ }^{2}$ \\ ${ }^{1}$ The Auroral Observatory, Institute of Physics, University of Tromsø, N-9037 Tromsø, Norway \\ ${ }^{2}$ University of Oslo, Institute for Theoretical Astrophysics, Box 1029 Blindern, N-0315 Oslo, Norway \\ ${ }^{3}$ University of Oslo, Physics Department, Box 1048 Blindern, N-0316 Oslo, Norway
}

Received: 5 April 2000 / Revised: 5 September 2000 / Accepted: 25 September 2000

\begin{abstract}
Low frequency electrostatic waves are studied in magnetized plasmas with an electron temperature which varies with position in a direction perpendicular to the magnetic field. For wave frequencies below the ion cyclotron frequency, the waves need not follow any definite dispersion relation. Instead a band of phase velocities is allowed, with a range of variation depending on the maximum and minimum values of the electron temperature. Simple model equations are obtained for the general case which can be solved to give the spatial variation of a harmonically time varying potential. A simple analytical model for the phenomenon is presented and the results are supported by numerical simulations carried out in a $2 \frac{1}{2}$-dimensional particle-in-cell numerical simulation. We find that when the electron temperature is striated along $\mathbf{B}_{0}$ and low frequency waves $\left(\omega \ll \Omega_{c i}\right)$ are excited in this environment, then the intensity of these low frequency waves will be striated in a manner following the electron temperature striations. High frequency ion acoustic waves $\left(\omega \gg \Omega_{c i}\right)$ will on the other hand have a spatially more uniform intensity distribution.
\end{abstract}

Key words: Ionosphere (plasma temperature and density) - Radio science (waves in plasma) Space plasma physics (numerical simulation studies)

\section{Introduction}

The propagation of low frequency electrostatic waves in magnetized plasmas represents a classic study in low temperature laboratory plasmas. In one of the most

\footnotetext{
* Present address: University of Oslo, Institute for Theoretical Astrophysics, Box 1029 Blindern, N-0315 Oslo, Norway

Correspondence to: P. Guio

e-mail: Patrick.Guio@phys.uit.no
}

versatile experimental set-ups, the Q-machine, extensive investigations were reported on the propagation of low frequency ion sound waves along an externally imposed magnetic field, i.e. in an essentially one dimensional geometry (Motley, 1975). Ideally, the conditions in the direction transverse to the magnetic field were assumed constant, but in reality the plasma density as well as the ion and electron temperatures can vary in these experiments and in similar ones. Also, it may be a rule rather than an exception that the electron as well as the ion temperatures are spatially inhomogeneous in naturally occurring plasmas out of equilibrium (Peñano etal., $2000)$. In the present study we investigate some consequences of a spatially varying electron temperature. The study is carried out in a slab geometry with a homogeneous magnetic field along the $z$-axis and $T_{e}=T_{e}(x)$. This electron temperature variation is assumed to be given a priori. We find, for instance, that in cases where the electron temperature is striated along $\mathbf{B}_{0}$ and low frequency waves $\left(\omega \ll \Omega_{c i}\right)$ are excited in this environment, for instance by a plasma instability, then the intensity of these low frequency waves will be striated in a manner following the electron temperature striations. High frequency ion acoustic waves $\left(\omega \gg \Omega_{c i}\right)$ will, on the other hand, have a spatially more uniform intensity distribution. These effects should be readily noticeable by, for instance, an instrumented space craft. The basic physical principles underlying the analysis are quite simple, and the phenomena addressed in the present work can have importance for the interpretation of, e.g. ionospheric or magnetospheric plasma phenomena.

\section{Analytical results}

For the present study we are primarily interested in waves with frequency well below the ion cyclotron frequency. However, this problem contrasts in an interesting way with its high frequency counterpart. Both cases are therefore discussed. In the linearized analysis used in the present study, a density gradient will be immaterial, since the linear sound velocity does not 
depend on the plasma density. The investigations are therefore mainly concerned with variations in the electron temperature.

\subsection{The low frequency case, $\omega<\Omega_{c i}$}

In this section we consider a simple analytical model which seems to account for the essential parts of our findings. The model assumes a vanishing ion temperature $T_{i}=0$ in the quasi-neutral limit where $n_{e} \approx n_{i} \equiv n$. The basic equations are the ion continuity and momentum equations as well as the $z$-component of the electron momentum equation where the electron inertia has been neglected. These equations are respectively, in their linearized form

$\frac{\partial n}{\partial t}+n_{0} \boldsymbol{\nabla} \cdot \mathbf{u}=0$

$M \frac{\partial \mathbf{u}}{\partial t}-e\left(-\nabla \phi+\mathbf{u} \times \mathbf{B}_{0}\right)=0$

$-e n_{0} \frac{\partial \phi}{\partial z}+\frac{\partial}{\partial z}\left[n T_{e}(x)\right]=0$

where $n$ and $\mathbf{u}$ are the density and velocity perturbations, $T_{e}$ is the electron temperature, $\phi$ the electrostatic potential $(\mathbf{E}=-\nabla \phi), \mathbf{B}_{0}$ the magnetic field, $e$ and $M$ the ion charge and mass, respectively. Assuming that the relevant frequencies are well below the ion-cyclotron frequency $\Omega_{c i}$, we iterate Eq. (2) in order to obtain the standard approximation for the ion velocity component perpendicular to the magnetic field

$\mathbf{u}_{\perp}=-\frac{\boldsymbol{\nabla}_{\perp} \phi \times \mathbf{B}_{0}}{B_{0}^{2}}-\frac{1}{B_{0} \Omega_{c i}} \frac{\partial \boldsymbol{\nabla}_{\perp} \phi}{\partial t}$,

The first term is the $\mathbf{E} \times \mathbf{B}$ drift velocity and the second term the ion polarization drift. With a little standard algebra and in the relevant two spatial dimensions $(x, z)$, we obtain a differential equation for the electrostatic potential

$\frac{e}{T_{e}(x)} \frac{\partial^{2} \phi}{\partial t^{2}}-\frac{1}{B_{0} \Omega_{c i}} \frac{\partial^{2}}{\partial t^{2}} \frac{\partial^{2} \phi}{\partial x^{2}}-\frac{e}{M} \frac{\partial^{2} \phi}{\partial z^{2}}=0$.

The $\mathbf{B}_{0}$-perpendicular component of the electron dynamics does not enter the analysis with the assumption of Boltzmann distributed electrons, see also a brief discussion in Appendix A. As mentioned, we here assume that the ion temperature is vanishing for simplicity. The results for $T_{i} \neq 0$ are not significantly different, as demonstrated in Appendix B.

For a homogeneous plasma with constant electron temperature $T_{e}$, we find the standard dispersion relation

$\omega^{2}=\frac{k_{z}^{2} C_{s}^{2}}{1+k_{x}^{2} C_{s}^{2} / \Omega_{c i}^{2}}$

for $\omega^{2} \ll \Omega_{c i}^{2}$, where $C_{s}=\sqrt{T_{e} / M}$ is the ion sound acoustic velocity and $C_{S} / \Omega_{c i}$ takes the role of an effective Larmor radius. The dispersion relation is shown in Fig. 1 (upper panel), together with a vectorial presentation of the group velocity $\mathbf{v}_{g}$, deduced from Eq. (6)

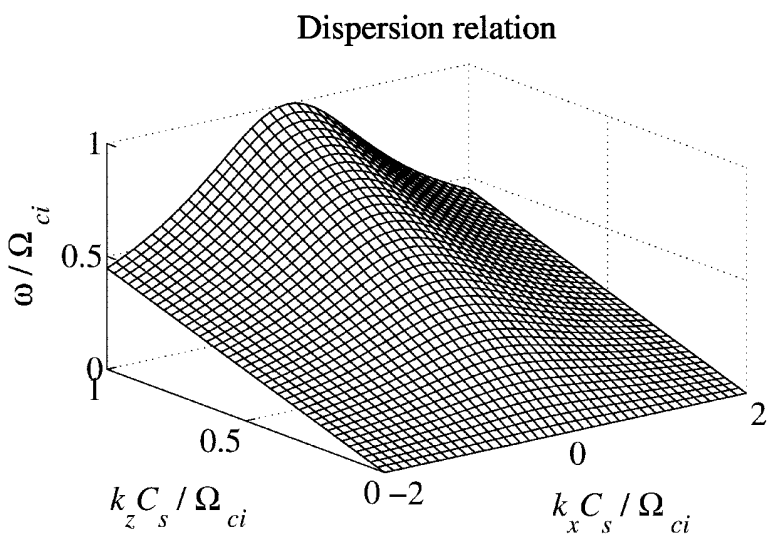

Group velocity $v_{g}$

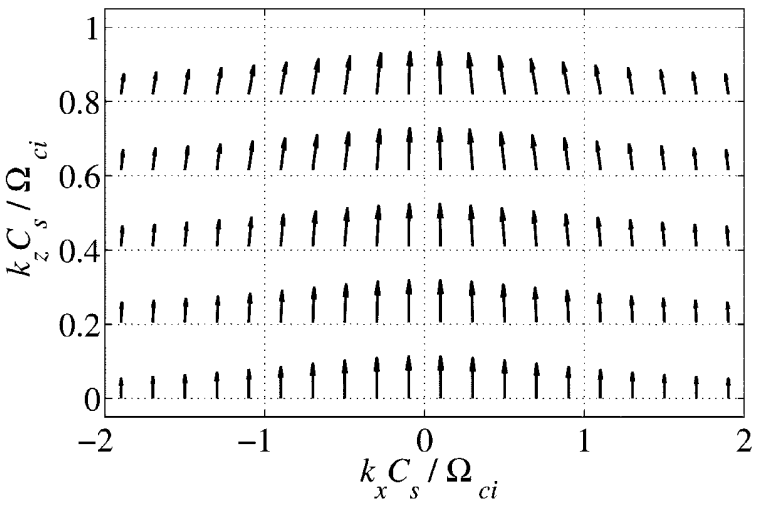

Fig. 1. In the upper panel, the dispersion relation $\omega=\omega\left(k_{x}, k_{y}\right)$ given by Eq. (6) is shown for $\omega<\Omega_{c i}$. In the lower panel, the corresponding group velocity $\mathbf{v}_{g}$. The field of the group velocity is represented by small arrows that give direction and relative magnitude. The results apply for $\omega^{2} \ll \Omega_{c i}^{2}$

(lower panel). We note that $\mathbf{k} \cdot \mathbf{v}_{g}=k_{z} C_{s} /\left(1+k_{x}^{2} C_{s}^{2} /\right.$ $\left.\Omega_{c i}^{2}\right)^{3 / 2}$, and the directions of $\mathbf{k}$ and $\mathbf{v}_{g}$ are different. In Fig. 2 we show the variation of the angle between the wave vector and the group velocity for varying wave vector components, $k_{x}$ and $k_{z}$.

Retaining the spatially varying electron temperature, we can still take the Fourier transform in time and in space, along the $z$-axis, to obtain

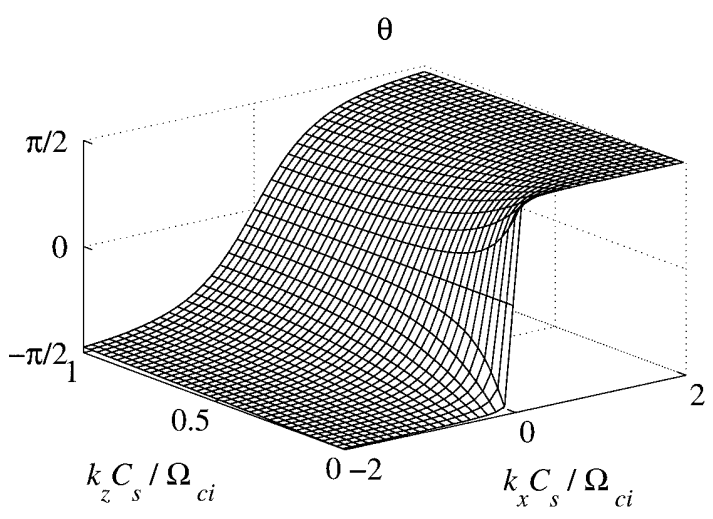

Fig. 2. The variation of the angle between the wave vector and the group velocity for varying wave vector components, $k_{x}$ and $k_{z}$. The point $\left(k_{x}, k_{z}\right)=(0,0)$ is singular. The results apply for $\omega^{2} \ll \Omega_{c i}^{2}$ 
$\frac{\mathrm{d}^{2} \phi}{\mathrm{d} x^{2}}=\left(\frac{M \omega^{2}}{T_{e}(x)}-k_{z}^{2}\right) \frac{\Omega_{c i}^{2}}{\omega^{2}} \phi$.

In the limit where we ignore the ion polarization drift, the left side of Eq. (7) vanishes, and the result is

$\frac{\omega^{2}}{k_{z}^{2}}=\frac{T_{e}(x)}{M}$.

Physically, Eq. (8) implies that in this limit each magnetic field line supports oscillations with the local sound velocity, irrespective of what happens at another field line. The system has a local, but no global dispersion relation. If excited externally, the system will support a continuum of phase velocities in the range determined by the minimum and maximum values of the electron temperature. In many ways this low frequency limit of magnetized plasmas has similarities with Langmuir oscillations with cold electrons in spatially varying plasma densities (Barston, 1964), and also with Alfvén waves propagating in inhomogeneous magnetic fields (Uberoi, 1972).

For a more general analysis of Eq. (7) it is advantageous to introduce the normalized variables $\gamma^{2} \equiv M \omega^{2} /\left(T_{0} k_{z}^{2}\right)$ and $\xi \equiv x \Omega_{c i} / C_{s}$, in terms of a reference temperature $T_{0}$. The reference temperature can be defined as $T_{0} \equiv \frac{1}{2}\left(T_{1}+T_{2}\right)$, where $T_{2}$ and $T_{1}$ are the maximum and minimum values the electron temperature attains. We find

$\frac{\mathrm{d}^{2} \phi}{\mathrm{d} \xi^{2}}=\left(\frac{T_{0}}{T_{e}(\xi)}-\frac{1}{\gamma^{2}}\right) \phi$

For later reference we define $k_{T_{j}}^{2} \equiv 1 / \gamma^{2}-T_{0} / T_{j}$ in the case where $\gamma^{2}<T_{j} / T_{0}$ and $1 / \lambda_{T_{j}}^{2} \equiv T_{0} / T_{j}-1 / \gamma^{2}$ otherwise, with $j=1,2$. The relation Eq. (9) can be interpreted as an operator equation with $\phi$ being the eigenfunction and $1 / \gamma^{2}$ the corresponding eigenvalue.

2.1.1 Monotonic electron temperature variation. Let us assume first that the variation in the electron temperature is monotonic, decreasing from $T_{2}$ at $\xi \rightarrow-\infty$ to $T_{1}$ at $\xi \rightarrow \infty$. For a given value of $\left(\omega, k_{z}\right)$, we require that Eq. (9) has physically acceptable solutions which are bounded at infinity. For the case where $\gamma^{2}>T_{2} / T_{0}$ this is not possible, since the solution for $\phi$ diverges either at $\xi \rightarrow \infty$ or $\xi \rightarrow-\infty$. In the case where $\gamma^{2}<T_{1} /$ $T_{0}<T_{2} / T_{0}$ we have solutions of Eq. (9) with $\phi \rightarrow \exp$ $\left(i k_{T_{1}} \xi\right)$ for $\xi \rightarrow \infty$, and $\phi \rightarrow \exp \left(i k_{T_{2}} \xi\right)$ for $\xi \rightarrow-\infty$. In the case where $\gamma^{2}=T_{e}(\xi) / T_{0}$ for some value of $\xi$, we find solutions for $\phi$ which decay as $\exp \left(-\xi / \lambda_{T_{1}}\right)$ when $\xi \rightarrow \infty$, and oscillate for $\xi \rightarrow-\infty$. Physically this result implies that the preselected value of the phase velocity $\omega / k_{z}$ must resonate with the natural sound velocity at a certain point to have such an acceptable solution. As long as such an internal resonance point exists, i.e. that $T_{1} / T_{0}<\gamma^{2}<T_{2} / T_{0}$, it is possible to obtain physically acceptable solutions for any combination of $\omega$ and $k_{z}$. For an arbitrary temperature profile $T_{e}(\xi)$, the solution for $\phi$ can only be obtained numerically for the general case. In Fig. 3 we show examples for numerical solutions, assuming an electron temperature described

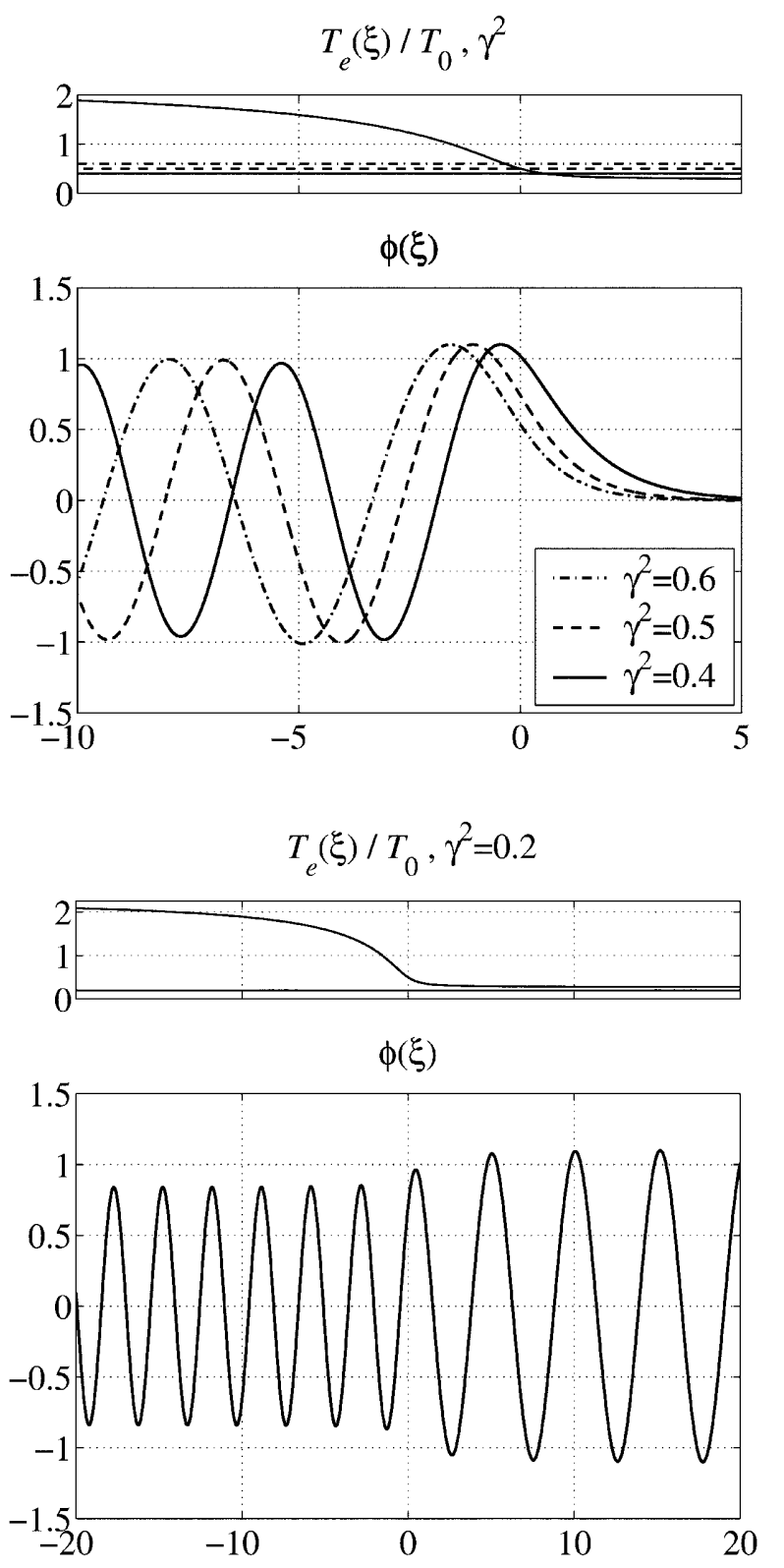

Fig. 3. Numerical solution of Eq. (9), assuming a monotonically decreasing electron temperature variation $T_{e}(\xi) / T_{0}=1 /(2+$ $\arctan (\xi))$. The first and third panels show the relative value of $\gamma^{2}$ compared to the temperature profile $T_{e}(\xi) / T_{0}$. The second panel presents solution for the electrostatic potential $\phi$ for $\gamma^{2}=0.4,0.5$ and 0.6 while the solution in the fourth panel has parameter $\gamma^{2}=0.2$

by $T_{0} / T_{e}(\xi)=2+\arctan (\xi)$ and in Fig. 4 , an electron temperature described by $T_{e}(\xi) / T_{0}=1-\frac{1}{2} \Delta+\Delta \exp$ $\left(-\xi^{2} / W^{2}\right)$. Some local solutions can however be found analytically. Assume for instance $T_{0} / T_{e}(\xi) \approx \xi$ in a certain interval, then we readily find $\phi=A i\left(\xi-1 / \gamma^{2}\right)$ locally, in terms of the Airy function $A i$.

2.1.2 Compact electron temperature variation. Let us next assume that the electron temperature variation is compact, first with a local enhancement with a maximum value $T_{2}$ at $\xi=0$, and $T_{e}(\xi) \rightarrow T_{1}$ for $|\xi| \rightarrow \infty$. For $\gamma^{2}>T_{2} / T_{0}$ there are no physically acceptable solutions. 

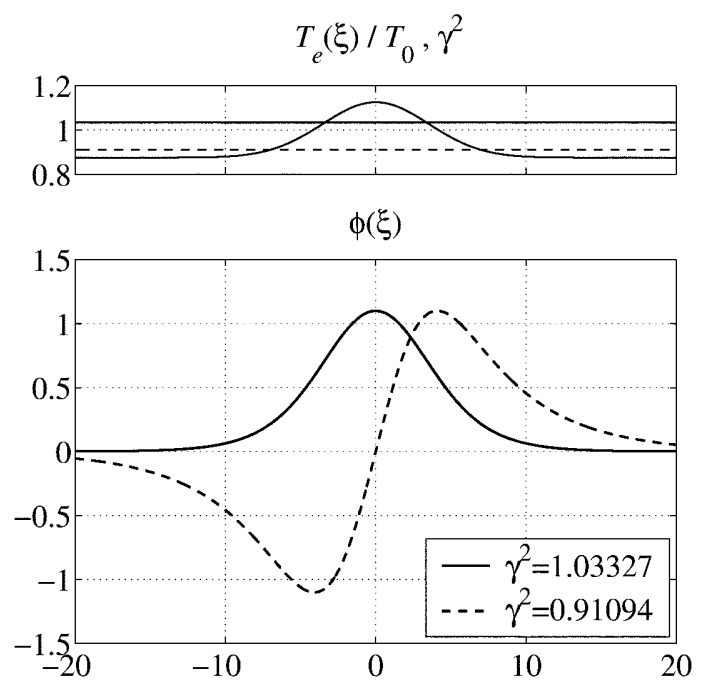

Fig. 4. Numerical solution of Eq. (9), assuming an electron temperature profile given by $T_{e}(\xi) / T_{0}=1-\Delta / 2+\Delta \exp \left(-\xi^{2} / w^{2}\right)$, with $w=5$ and $\Delta=-1 / 4$. The wave guide mode solutions for the potential are plotted for $\gamma^{2}=1.03327$ and $\gamma^{2}=0.91094$

In the case $\gamma^{2}<T_{1} / T_{0}$, we have solutions of Eq. (9) with $\phi \rightarrow \exp \left(i k_{T_{1}} \xi\right)$ for $|\xi| \rightarrow \infty$, having the form of "radiating" solutions. If on the other hand, $T_{1} / T_{0}<$ $\gamma^{2}<T_{2} / T_{0}$, it is possible to obtain wave guide modes for selected values of $\gamma$. Examples are shown in Fig. 4. The ratio between the two phase velocities is approximately 0.93 . The basic, universal, features are the evanescent solutions at large $|\xi|$, and the possibility for oscillations around the maximum temperature region at $\xi \approx 0$. Note that a local analytical solution can be obtained by assuming spatial variations of the temperature as $T_{0} / T_{e}(\xi) \approx \xi^{2}$.

The physical reason for the Airy function-like solution is easy to explain. Taking $\gamma^{2}=1$, we have a resonance with the local sound velocity at $\xi=0$ with $k_{\xi}=0$. For the local phase fronts at $\xi<0$ to follow those at $\xi=0$, we require that the local component of the phase velocity parallel to the magnetic field should be equal at all $\xi$. Since here $C_{s}$ is increasing for $\xi<0$, we must require a local wave number $k_{\xi} \neq 0$ here, according to Eq. (6) to compensate the increase in $T_{e}$. On the other hand, for $\xi>0$ there is no possibility to have local phase velocities matching that at $\xi=0$, and the solution for $\phi$ must be evanescent.

If the variation of $T_{e}(\xi)$ can be characterized by a single length scale $\mathscr{L}$, the continuum limit is recovered for $\mathscr{L} \rightarrow \infty$. Here, the density of the eigenvalues becomes larger with $\mathscr{L}$ increasing and form a continuum in the limiting case, irrespective of the actual functional form of $T_{e}(\xi)$.

Using Eq. (9) we find, by a simple manipulation the relation

$\frac{\mathrm{d}}{\mathrm{d} \xi}\left(\frac{\mathrm{d} \phi}{\mathrm{d} \xi}\right)^{2}=\left(\frac{T_{0}}{T_{e}(\xi)}-\frac{1}{\gamma^{2}}\right) \frac{\mathrm{d} \phi^{2}}{\mathrm{~d} \xi}$.

From Eq. (10) we find, for instance, that at a zero crossing of $\phi$ we have a local extremum of $(\mathrm{d} \phi / \mathrm{d} \xi)^{2}$, in general, irrespective of $T_{e}(\xi)$.
Parts of the foregoing discussion assumed a compact local temperature enhancement. The discussion for a local depletion is entirely similar, with the result that "radiating" solutions at $|\xi| \rightarrow \infty$ will here exist also for the case where $\gamma^{2}=T_{e}(\xi) / T_{0}$ for some value of $\xi$. An example is shown in Fig. 5.

\subsection{The high frequency case, $\omega>\Omega_{c i}$}

Within the simple fluid model discussed here, we might also consider the case of waves with frequency well above the ion cyclotron frequency. The plasma is still magnetized and can support the spatially varying electron temperature, but due to the assumed high frequency we can consider the ions as unmagnetized. The basic equations are trivially obtained from Eqs. (1)-(3) but by omitting the magnetic force $\mathbf{u} \times \mathbf{B}$. The resulting differential equation for the potential becomes

$\frac{\mathrm{d}^{2} \phi}{\mathrm{d} x^{2}}=\left(k_{z}^{2}-\frac{M \omega^{2}}{T_{e}(x)}\right) \phi$

which is basically the same as Eq. (7), apart from a difference in a numerical coefficient and an important change in sign. The physical arguments are readily reformulated to account for this case as well. The resulting radiation pattern is similar to the one anticipated by Huld et al. (1990) from the coupling between a beam mode and the background acoustic mode in a plasma, where the injected ion beam has a finite cross-section.

\section{Numerical results}

We carried out a numerical investigation in order to study elements of the analytical results. The numerical simulations are carried out by using a particle-in-cell (PIC) code (Dawson, 1983; Birdsall and Langdon,
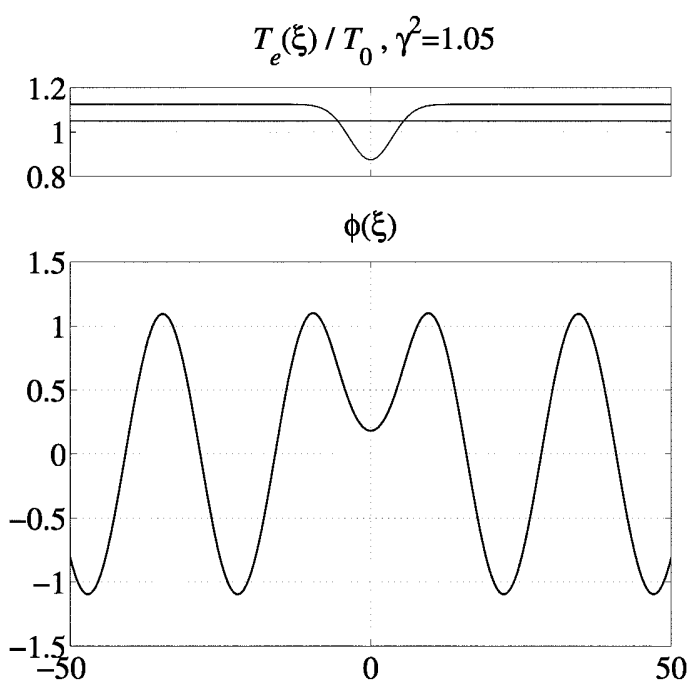

Fig. 5. Numerical solution of Eq. (9) assuming a localized depletion in electron temperature given by $T_{e}(\xi) / T_{0}=1+\Delta / 2-\Delta$ $\exp \left(-x^{2} / w^{2}\right)$ with the parameters $w=5$ and $\Delta=1 / 4$. A solution has been calculated for $\gamma^{2}=1.05$ 
1985). Its basic elements is described elsewhere (Børve et al., 2000). We use typically $6.10^{6}$ simulation particles. In order to improve the signal to noise ratio in the low amplitude, linear fluctuations analyzed in this study, we average over 10 realizations, all with the same bulk plasma conditions, but with different initialization of the random number generator for the initial position of the particles. The essential feature of the code is the assumption of the electrostatic potential of the electrons being Boltzmann distributed at all times, in the present case at the temperature $T_{e}=T_{e}(x)$. This simplification is achieved at the expense of a nonlinear Poisson equation. The code is in $2 \frac{1}{2}$ dimensions, i.e. it allows the ions to perform the full gyro motion, but assumes that all spatial variations are restricted to the $(x, z)$-plane. With these numerical restrictions, waves propagating in the direction of the electron diamagnetic drift, i.e. perpendicular to the $(x, z)$-plane of the simulation, will not be manifested. We use $T_{2} / T_{1}=6$ and $T_{1} / T_{i}=3$, in terms of the maximum and minimum values of the electron temperature, $T_{1}$ and $T_{2}$, for a constant ion temperature, $T_{i}$. Axes are in units of the ion Debye length $\lambda_{D i} \equiv \sqrt{\varepsilon_{0} T_{i} / e^{2} n_{0}}$ in all cases.

\subsection{The low frequency case, $\omega<\Omega_{c i}$}

3.1.1 Monotonic temperature variations. First we consider frequencies $\omega<\Omega_{c i}$. In Fig. 6 we show the results for a harmonically varying density perturbation of $10 \%$ introduced at $z=0$, in a plasma where the electron temperature varies monotonically along the $x$-axis as $T_{e}(x) \sim \arctan (x / \Delta)$ with $\Delta=7.125$. The density perturbation is parallel to the $x$-axis at $z=0$ and has a Gaussian shape centred in the bounded $x$-direction. The conspicuous features are readily noted. The phase fronts, which are parallel by construction at the position $z=0$, are tilting for $z>0$ due to the variation with $x$ of the phase velocity. Due to the resulting change in direction of the group velocity, see Fig. 1, the wave-field moves with the group velocity towards the region of enhanced electron temperature. Figure 6 shows two such numerical simulations, with two $x$-positions for the maximum of the density perturbations with respect to the electron temperature variation. The excitation at the boundary is not the one corresponding to a solution of Eq. (9), but we note that the perturbation seems to evolve towards such a solution. In particular we recognize the local maximum of the fluctuating potential in the vicinity of the steepest electron temperature gradient.

3.1.2 Localized temperature variations. In continuation of the foregoing results we carried out a simulation with a localized enhancement of the electron temperature described by a Gaussian $T_{e}(x) \sim \exp \left(-x^{2} / 2 \Delta^{2}\right)$, with parameter $\Delta=11.875$. In Fig. 7 we show the variations along the magnetic field of the wave pattern at a fixed position $x=33$. The waves are excited by a harmonic $10 \%$ density excitation at $z=0$. The solid line corresponds to the case where the excitation has a profile along the $x$-axis as $\delta n \sim \exp \left(-x^{2} / \Delta^{2}\right)$ and the dashed
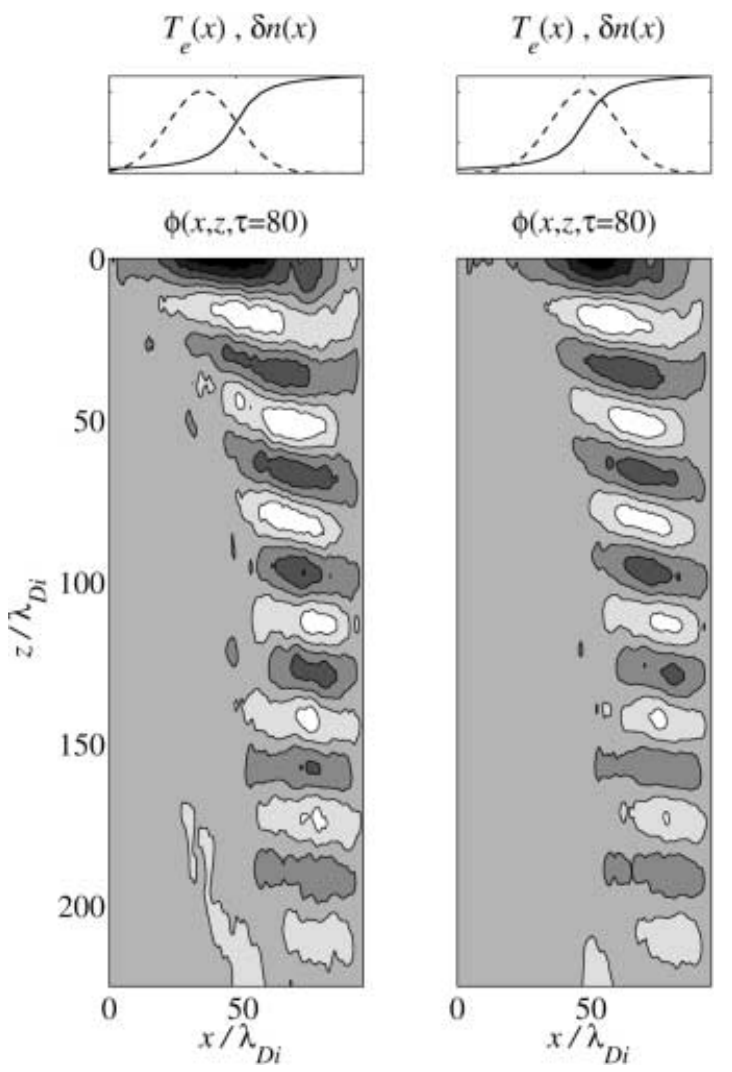

Fig. 6. Numerical simulation of the potential $\phi$ that results from a harmonically varying density perturbation introduced at $z=0$. The electron temperature $T_{e}$ is assumed to vary as arctan in the $x$-direction (solid line in the upper panel). In the right panel, the density perturbation $\delta n$ is maximum in the middle and decreases as a Gaussian (dashed line in the upper panel) while in the left panel, the density perturbation $\delta n$ is moved half way left. The magnetic field points in the $z$-direction. The applied frequency is $\omega=0.2 \pi \omega_{p i}$ while $\Omega_{c i}=\omega_{p i}$ and $\tau$ represents time normalized to the ion time scale $1 / \omega_{p i}$

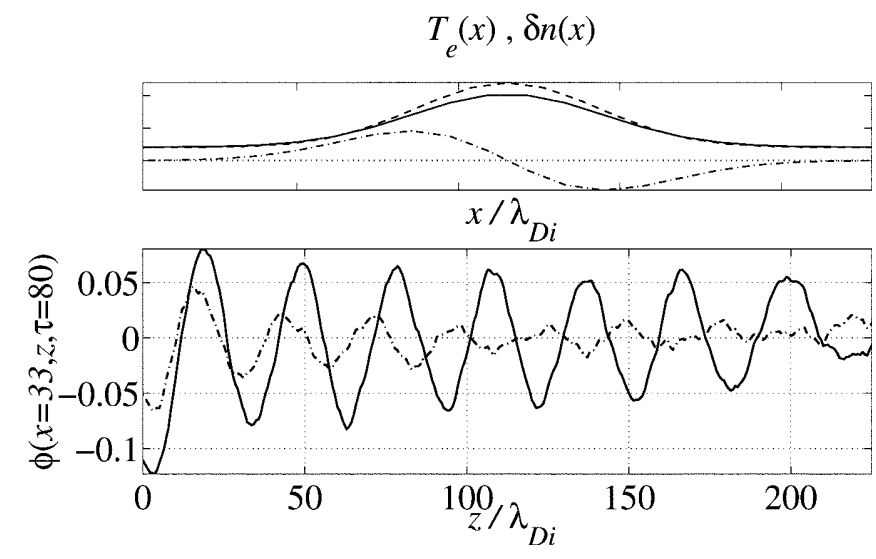

Fig. 7. Numerical simulation of the potential variation along the $z$-axis, i.e. parallel to the magnetic field, for a fixed $x$. The electron temperature enhancement is localized as a Gaussian in the $x$-direction, i.e. transverse to the magnetic field, for two wave guide modes. In the upper panel and in arbitrary units, we sketched the temperature profile (dashed line) as well as the two different density perturbations $\delta n \sim \exp \left(-x^{2} / \Delta^{2}\right) \quad$ (solid line) and $\delta n \sim x \exp \left(-x^{2} / \Delta^{2}\right)$ (dash-dot line). The dotted line is the reference density level without perturbation. The applied frequency is $\omega=0.2 \pi \omega_{p i}$ while $\Omega_{c i}=\omega_{p i}$ 
line to the case of a profile as $\delta n \sim x \exp \left(-x^{2} / \Delta^{2}\right)$. We recognize two different phase velocities corresponding to the two different waveguide modes being excited. The ratio of the two phase velocities is approximately 0.89 .

As another feature of the localized electron variation we consider the same conditions as in Fig. 8, but now let the density perturbation be given as $\delta n \sim \exp \left(-x^{2} / \Delta^{2}\right)$ and $\delta n \sim 1-\exp \left(-x^{2} / \Delta^{2}\right)$ for the two cases shown by solid and by dashed lines, respectively. The interesting point is here that the phase velocity of the two modes are virtually identical, but they have somewhat different damping length, even though the two curves are obtained at the same spatial position and at the same excitation frequency. The reason for the difference can be understood by the result from Fig. 6. Due to the variation in phase velocity the phase fronts are tilted at the positions with the strongest gradient in electron temperature. Also in this case we have a flow of wave energy by the group velocity, which here enters the region of density enhancement from both sides. In the first excitation, there is little wave energy outside the high electron temperature region, and in this case the wave evolution is entirely dominated by the local ion Landau damping. In the latter case the Landau damping is still effective, but part of the energy loss is compensated by energy inflow from the outer regions which in this case are excited stronger.

\subsection{The high frequency case, $\omega>\Omega_{c i}$}

Figure 9 shows the variations along the magnetic field as well as a two-dimensional picture of the waves for the same temperature and excitation conditions but with

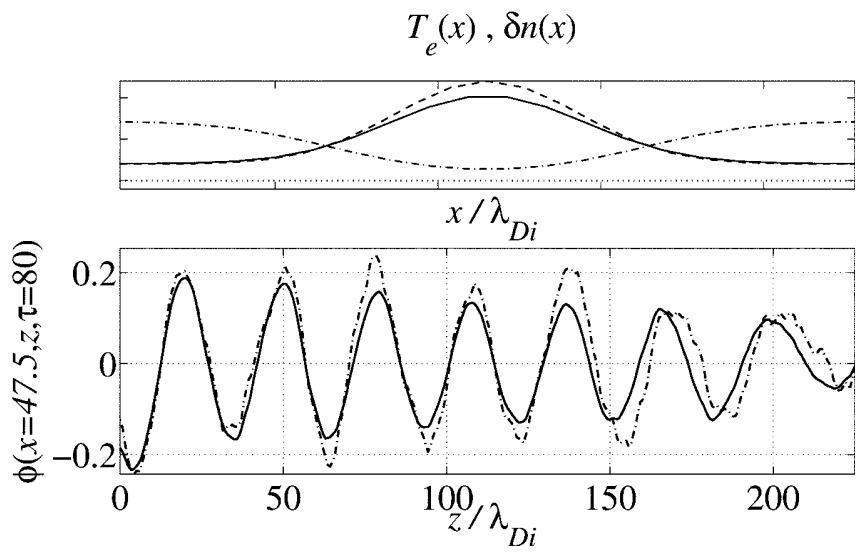

Fig. 8. Numerical simulation of the potential variation along the $z$ axis, i.e. parallel to the magnetic field, for a fixed $x$. The electron temperature enhancement is localized as a Gaussian in the $x$-direction, i.e. transverse to the magnetic field, for two different excitations. In the upper panel and in arbitrary units, we sketched the temperature profile (dashed line) as well as the two different density perturbations, $\delta n \sim \exp \left(-x^{2} / \Delta^{2}\right)$ (solid line) and $\delta n \sim \alpha-\exp \left(-x^{2} / \Delta^{2}\right)$ (dash-dot line). The dotted line is the reference density level without perturbation. In the lower panel, the corresponding variations of potential, the dash-dot line curve has been multiplied by approximately 4 for readability. Note the difference in damping distance. The applied frequency is $\omega=0.2 \pi \omega_{p i}$ while $\Omega_{c i}=\omega_{p i}$
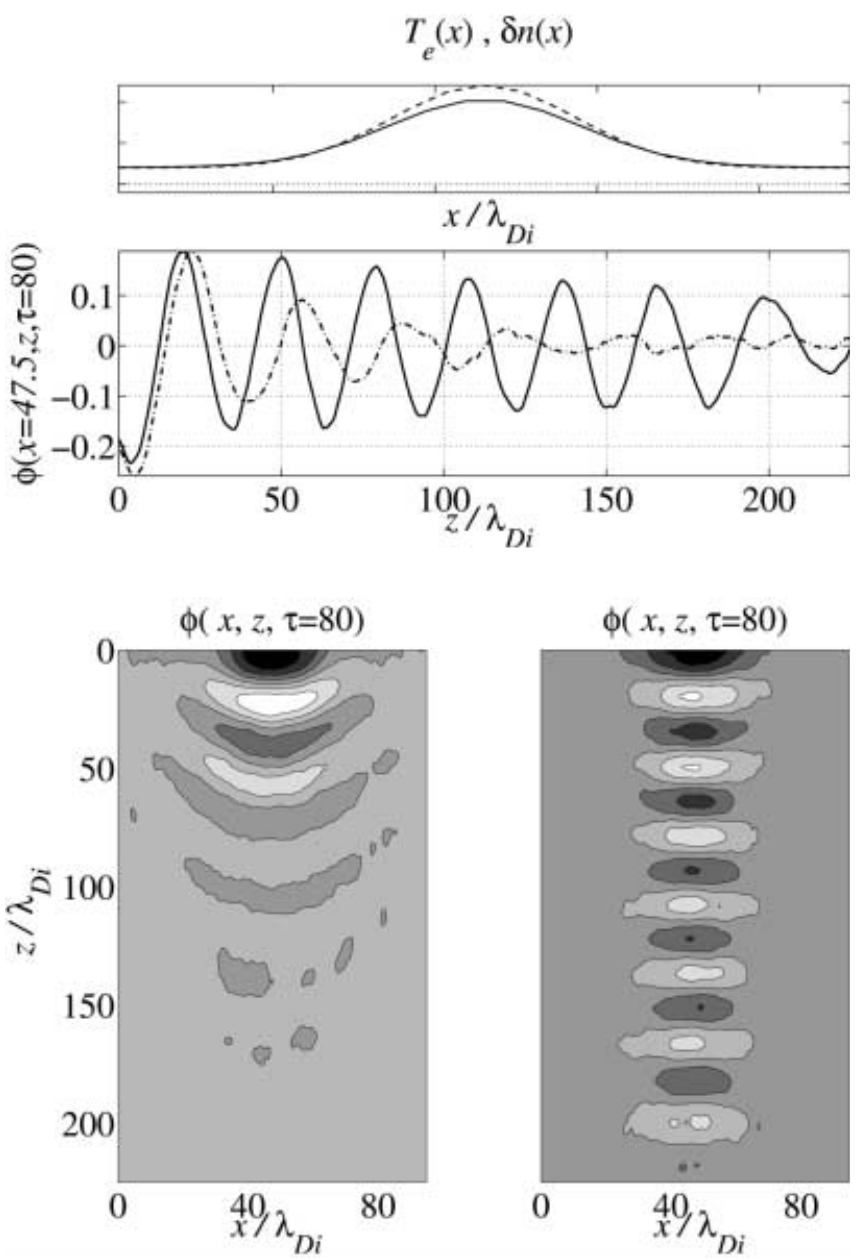

Fig. 9. The second panel from top shows numerical simulation of the potential variation along the $z$-axis, i.e. parallel to the magnetic field, for a fixed $x=47.5$. The electron temperature enhancement is localized as a Gaussian in the $x$-direction, the excitations are identical. In the upper panel and in arbitrary units, we sketched the temperature profile (dashed line) as well as the density perturbations $\delta n \sim \exp \left(-x^{2} / \Delta^{2}\right)$ (solid line). The dotted line is the reference density without perturbation. The difference is the magnetic field strength. The solid line is for magnetized while the dashed line is for a very weakly magnetized. The two lowest panels present the variations in two dimensions of the electrostatic potential. We have $\omega=0.2 \pi \omega_{p i}$ in both cases while $\omega_{c i} / \Omega_{p i}=0.05$ and $\omega_{c i} / \Omega_{p i}=1$ in the lowest left and right panels respectively

different magnetic field strength, i.e. in one case with $\omega<\Omega_{c i}$ and in the other case $\omega>\Omega_{c i}$. The conspicuous difference between the two cases is evidently the difference in damping. For the case where $\omega>\Omega_{c i}$, the explanation of the rapid deployment of the wave amplitude is quite simple; due to the $x$-variation of the ion sound speed, the phase fronts curve to become convex, and the local group velocity obtains an $x$-component, which leads to a depletion of the energy density on axis, in excess of what would be obtained due to the linear ion acoustic Landau damping alone. For the case where $\omega<\Omega_{c i}$, the situation is the opposite. With hindsight, it may actually seem surprising that results from laboratory experiments often show a remarkable agreement with predictions from a simple 
theory based on homogeneous, uniform plasmas. Usually such a good agreement is obtained by fitting one or more free parameters, which in a way serves to "hide" the effects of inhomogeneities.

To make the effects discussed in this section more conspicuous, we kept the applied frequency constant (and thereby the wavelength $\lambda=2 \pi C_{s} / \omega$ ) and varied the externally applied magnetic field.

\section{Nonlinear waves}

The foregoing analysis was based on a linear approximation for ion acoustic waves. A complete analysis of the nonlinear evolution of these waves for the present geometries is outside our scope. Basically, we expect low frequency ion acoustic waves to steepen towards breaking, as simple waves in fluids (Kadomtsev and Karpman, 1971; Whitham, 1974).

It seems, however, that one particular nonlinear effect can be readily discussed on the basis of the linear results. The problem we have in mind is concerned with the case where the wave frequency, i.e. the fundamental frequency $\omega$ is below the ion cyclotron frequency, $\omega<\Omega_{c i}$, while on the other hand all harmonics are higher, $n \omega>\Omega_{c i}$ for $n>1$. This implies that as the wave steepens, it generates higher harmonics, but these harmonics will have properties different from the fundamental. In particular, the higher harmonics will be able to couple to the plasma surrounding the high electron temperature channel, as in Fig. 9, and thereby delay or even inhibit the wave breaking. In Fig. 10 we show the evolution of the wave field for the case where $\omega=0.1 \pi \omega_{p i}$ with $\Omega_{c i}=\omega_{p i} / 2$, for amplitudes increasing from $\delta n=0.1 n_{0}$ to $0.5 n_{0}$ by step of $0.1 n_{0}$. In Fig. 11 we have one example of the $(x, z)$-variations of the electrostatic potential for the amplitude $\delta n=0.5 n_{0}$. The $x$-variation of the electron temperature and the density perturbation at $z=0$ are the same as used in for instance Fig. 9. We see a rather complicated deformation of the phase fronts, clear signs

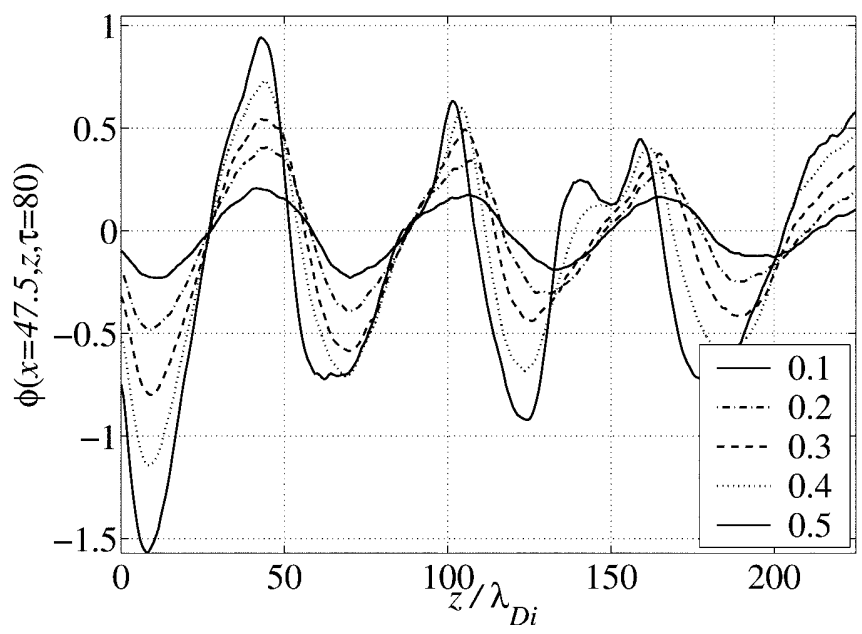

Fig. 10. The evolution of the electrostatic potential along the $z$-axis for increasing amplitudes $\delta n$ from $0.1 n_{0}$ to $0.5 n_{0}$. Here, we have $\Omega_{c i}=\omega_{p i} / 2$ while the applied frequency is $\omega=0.1 \pi \omega_{p i}$
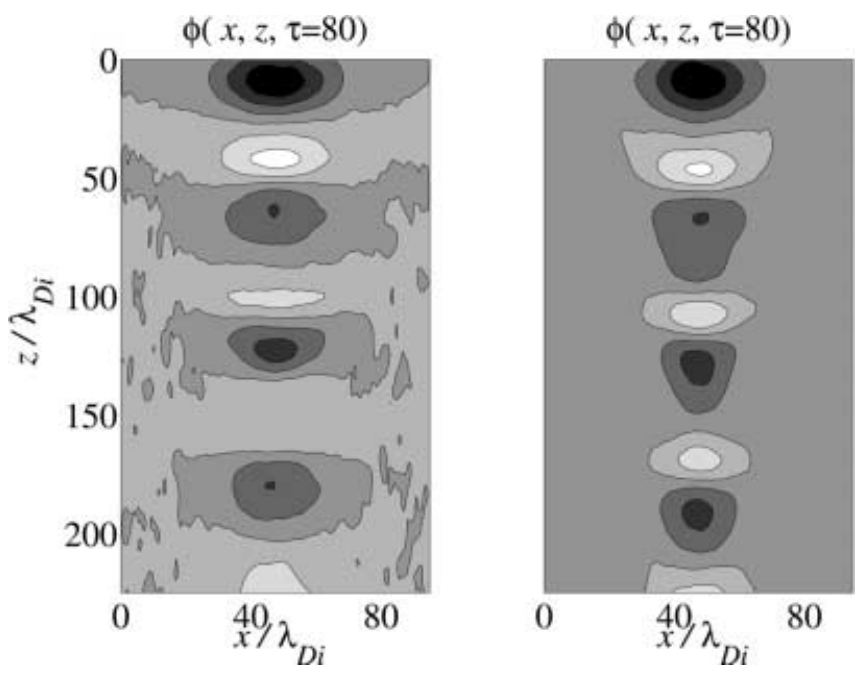

Fig. 11. The $(x, z)$-variation of the electrostatic potential for the amplitude $\delta n=0.5 n_{0}$ is shown for $\Omega_{c i}=\omega_{p i} / 2$ in the left panel and for $\Omega_{c i}=\omega_{p i}$ in the right panel

of harmonic generation, and also a systematic decrease of the phase velocity for increasing amplitudes.

If we now increase the magnetic field in such a way that $\Omega_{c i}=\omega_{p i}$, implying that the first and second harmonics of the applied frequency are now below the ion cyclotron frequency. An example for the wave evolution is shown in Fig. 12, for the case of maximum amplitude in Fig. 10. In this case we find a quite standard, almost text-book like, steepening and formation of $N$-waves, as for weakly damped one-dimensional nondispersive waves. The $(x, z)$-variation of the electrostatic potential for this amplitude is shown in Fig. 11. In the evolution of the harmonics of the fundamental frequency, the wave damping is competing with the growth of the harmonic content away from the plasma boundary at $z=0$. To lowest approximation, the $n$-th harmonic increase (Blackstock, 1962; Pécseli, 1985) as $\sum_{n}(2 / n \sigma) J_{n}(n \sigma) \sin (n(\omega t-k z))$ with normalized dis-

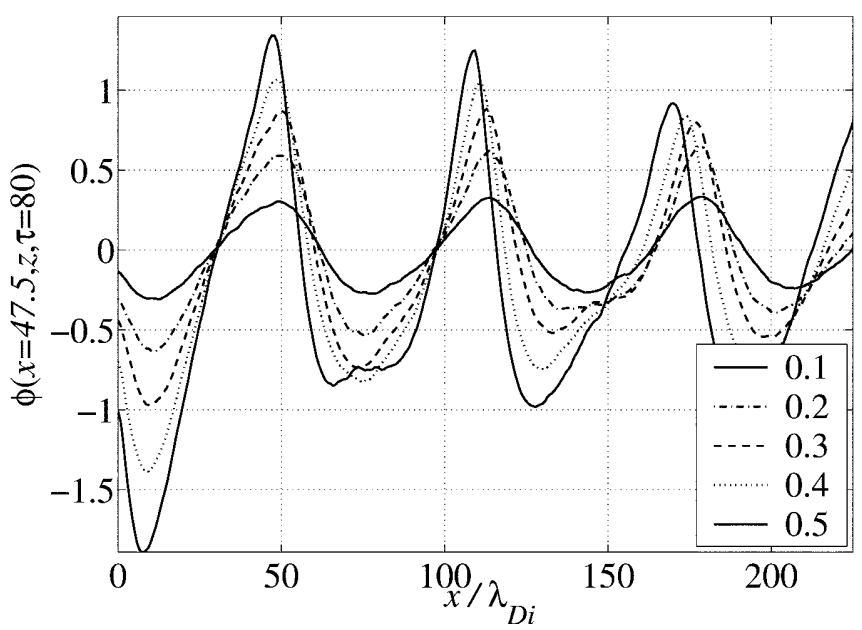

Fig. 12. The evolution of the electrostatic potential along the $z$-axis for perturbation amplitudes increasing in units of $0.1 \delta n / n_{0}$, with $\Omega_{c i}=\omega_{p i}$, and applied frequency $\omega=0.1 \pi \omega_{p i}$ 
tance, $\sigma \equiv z\left(\omega / C_{s}\right)\left(\delta n / n_{0}\right)$, expressed in terms of the Bessel functions $J_{n}$. This solution (often termed the Fubini solution) accounts for the lowest order nonlinearity, up to a relative applied amplitude in the excitation of approximately $0.2-0.3$ in Figs. 10 and 12 . For larger amplitudes we note an enhanced initial damping and an increasing asymmetry between the positive and negative parts of the wave. In this large amplitude limit, the particles trapped in the first half period of the oscillations escape as a weak beam in the second half, in a way similar to the one discussed by Sato et al. (1976). The wave amplitude is then diminished by an amount corresponding to the energy carried by these particles.

The conclusion of this analysis in this section is almost self evident; as long as the fundamental and first few harmonics are below the ion cyclotron frequency we may in effect consider the wave guide modes as almost one dimensional nondispersive waves also in their nonlinear evolution. If, however, the higher harmonics are above $\Omega_{c i}$, the coupling to the surrounding plasma effectively depletes the wave energy to delay the breaking. The observed change in phase velocity can, seemingly, not be explained simply within this model.

In support of our interpretation of the numerical results summarized here we draw attention to the reduced damping of the nonlinear waves in the case where $\omega \ll \Omega_{c i}$, compared in Figs. 10 and 12. This is consistent with the expected reduction in loss of energy from the wave harmonics to the plasma surrounding the high temperature channel.

We have also studied a linearly unstable case where an ion beam is injected from one end of the plasma along the magnetic field, in conditions where the electron temperature is increased in the central part of the spatial domain, as in Figs. 7 and 8. We considered two cases, one with $\Omega_{c i}=\omega_{p i}$ and another where $\Omega_{c i}=0.05 \omega_{p i}$. Also in these cases we observed a clear ducting of the wave energy, but point out that the interpretation of these cases is complicated by the linear instability conditions being electron temperature dependent.

\section{Conclusions}

We described results from investigations of low frequency ion sound waves in magnetized plasmas with a spatially varying electron temperature. In the linearized analysis used in the present study, a density gradient will be immaterial, since the linear sound velocity does not depend on the plasma density. If the present analysis is generalized to the full three dimensional magnetized case, the situation is different, since electrostatic drift waves can then be excited with properties determined by the combined effects of the scale-lengths for density and electron temperature (Kadomtsev, 1965). These waves will, however, be propagating in a direction perpendicular to the wave-field considered in this study.

We demonstrated by direct numerical PIC-simulations that the basic features of the observations in these simulations can be described adequately by a simple differential equation, which at low frequencies, $\omega<\Omega_{c i}$, contains the essential features with the exception of the ion Landau damping. We demonstrated the existence of a wave guide mode, and also illustrated some consequences of the anisotropy of the group velocity at $\omega<\Omega_{c i}$. Thus, if the electron temperature is striated along $\mathbf{B}_{0}$ and low frequency waves $\left(\omega \ll \Omega_{c i}\right)$ are excited in this environment, for instance by a plasma instability, then the intensity of these low frequency waves will be striated in manner following the electron temperature. High frequency ion acoustic waves $\left(\omega \gg \Omega_{c i}\right)$ will on the other hand have a spatially more uniform intensity distribution. These effects should be readily noticeable by for instance an instrumented space craft. Also, we expect our results concerning the nonlinear evolution of a wave in Sect. 4 and the dependence of the harmonic spectrum on the ratio $\omega / \Omega_{c i}$ to have relevance for the understanding of the nonlinear saturation of low frequency instabilities in magnetized plasmas. As an example relevant for space plasmas, we can refer to observations of low frequency ion-ion beam instabilities in the solar wind (Feldman et al., 1973).

When an efficient mechanism for the transfer of ion acoustic wave energy to the electrons can be found, the observations of entrainment of wave energy into a localized region by the anisotropy of the group velocity allows for the possibility of a nonlinear self-focusing of ion acoustic waves. The mechanism for the process is self evident (Kadomtsev and Karpman, 1971), and will be effective only for low frequency waves, $\omega<\Omega_{c i}$.

The results summarized have applications to many physical phenomena, since temperature gradients in magnetized plasmas can occur in a variety of naturally occurring conditions in the Earth's ionosphere (Nilsson et al., 1998) as well as in the Solar corona (Drago et al., 1991). Gradients or striations in the electron temperature of the ionospheric plasma can also be induced artificially by ionospheric heating experiments, using intense radio waves (Das and Fejer, 1979; Dysthe et al., 1983). Ion acoustic waves propagating in such an environment will be expected to be subject to the evolutions described in the present work, where we considered the electron temperature variation to be given a priori.

Localized, ducted, low frequency ion acoustic waves have been observed by instrumented space-craft, as reported by for instance Wahlund et al. (1994a, b, 1998), and we believe such observations deserve closer scrutiny in the light of the models for wave evolutions discussed in this study.

In order to produce clear illustrative results we chose large ranges of variation for the electron temperatures in our analysis. The basic physical properties are, however, unaffected by the actual values of the temperature ranges, and our basic analytical results can readily be applied to given conditions. We might add that the basic physical arguments are quite general and applies equally well to, for instance, shallow water waves propagating over ducted surfaces (Whitham, 1974). 


\section{Appendix A}

It might be appropriate to add a few comments concerning the absence of the $\mathbf{B}_{0}$-perpendicular electron dynamics in the analysis. Ignoring electron inertia, as usual for low frequency ion acoustic waves, we have

$e n \nabla \phi-\nabla\left(n T_{e}\right)+e n \mathbf{u} \times \mathbf{B}_{0}=0$

As an illustration we first take the case where $T_{e}=$ constant, but the unperturbed plasma has a density gradient in the direction perpendicular to $\mathbf{B}_{0}$, i.e. $n_{0}=n_{0}(x)$. For the $\mathbf{B}_{0}$-parallel component of Eq. (12), we have Eq. (3), which gives the Boltzmann distributed electrons $n=n_{0}(x) \exp \left(e \phi / T_{e}\right)$. By insertion of this relation into Eq. (12) we find the component perpendicular to $\mathbf{B}_{0}$ as $e n_{0} \mathbf{u} \times \mathbf{B}_{0}=T_{e} \boldsymbol{\nabla}_{\perp} n_{0}$, giving $u_{y}=\left(T_{e} / e B_{0}\right) \mathrm{d} \ln n_{0}(x) / \mathrm{d} x$, which is nothing but the electron diamagnetic drift associated with the unperturbed plasma density gradient. The equations have not been linearized to obtain this result. The electron density can be fluctuating as well as the plasma potential, but there will not be any electron fluid velocity component in the direction perpendicular to $\mathbf{B}_{0}$. We have an exact cancellation of the fluctuations in the electron diamagnetic drifts and the $-\nabla \phi \times \mathbf{B}_{0} / B_{0}^{2}$-drifts associated with the fluctuations in plasma density. We now take $n_{0}=$ const. and $T_{e}=T_{e}(x)$. The $\mathbf{B}_{0}$-parallel component Eq. (12) is unaffected, giving again the Boltzmann distribution of electrons now with a varying electron temperature. Using this in Eq. (12), we find again without any linearization that $u_{y}=\left(1-e \phi / T_{e}(x)\right)\left(T_{e}(x) / e B_{0}\right)$ $\mathrm{d} \ln T_{e}(x) / \mathrm{d} x \equiv\left(1-\ln n / n_{0}\right)\left(T_{e}(x) / e B_{0}\right) \mathrm{d} \ln T_{e}(x) / \mathrm{d} x$. In this case there is a fluctuating component of the bulk electron velocity, but its direction is here along the phase fronts of the ion acoustic waves, and therefore it does not contribute to any charge separations.

\section{Appendix B}

We considered the modifications of the results in Sect. 2 due to finite ion temperatures, and included for generality also a collision frequency $v$ for ions colliding with a neutral component. Rather than Eq. (2) we now have

$n M \frac{\partial \mathbf{u}}{\partial t}=-e n\left(\nabla \phi-\mathbf{u} \times \mathbf{B}_{0}\right)-\nabla\left(n T_{i}\right)-n M v \mathbf{u}$.

After some simple algebra, we find the modifications in the expressions for the ion velocity due to the finite ion pressure, and we end up with a new differential equation for the electrostatic potential

$$
\begin{aligned}
& \left(\frac{k_{z}^{2}}{M(\omega+i v)}\left[T_{e}(x)+T_{i}(x)\right]-\omega\right) \phi \\
& \quad+\frac{T_{e}(x)(\omega+i v)}{M\left(\Omega_{c i}^{2}-(\omega+i v)^{2}\right)} \frac{\mathrm{d}^{2}}{\mathrm{~d} x^{2}}\left(\phi\left[1+\frac{T_{i}(x)}{T_{e}(x)}\right]\right)=0 .
\end{aligned}
$$

Basically, the finite ion pressure adds to the electron pressure, but in addition $T_{i} \neq 0$ has some other consequences by changing the character of the differential equation for the linearized potential. In order to apply a fluid model, as done here, we implicitly assume $T_{i} / T_{e} \ll 1$, implying that the new terms appearing in Eq. (14) are small. With $v \neq 0$ we have now complex eigenvalues for the equation, but this is merely describing the wave damping due to the collisions.

With a little work, the simple results for $T_{i}=0$, Eqs. (9) and (10) can be generalized somewhat. Assuming as before that the electrostatic potential varies only in the $z$ - and $x$-directions, we can obtain the full ion velocity from the linearized equations, and ultimately find the relation

$\frac{\mathrm{d}^{2} \phi}{\mathrm{d} x^{2}}=\left(\omega^{2}-\Omega_{c i}^{2}\right)\left(\frac{k_{z}^{2}}{\omega^{2}}-\frac{M}{T_{e}(x)}\right) \phi$,

or in dimensionless units $\frac{\mathrm{d}^{2} \phi}{\mathrm{d} \xi^{2}}=\left(\Omega^{2}-1\right)\left(\frac{1}{\gamma^{2}}-\frac{T_{0}}{T_{e}(\xi)}\right) \phi$,

now with the two new dimensionless parameters $\Omega \equiv \omega / \Omega_{c i}$ and $1 / \gamma^{2} \equiv\left(T_{0} / M\right)\left(k_{z} / \omega\right)^{2}$. For the simple case with a simple temperature proportionality, $T_{e}(x)=\alpha T_{i}(x)$, with $\alpha=$ const. and $v=0$, the result Eq. (14) is an almost trivial modification of Eq. (15). For the sake of simplicity, the discussions in Sect. 2 are based on the simpler case with $T_{i}=0$.

These equations contain the foregoing results of Eqs. (9) and (11) as special limits, but the derivation may not be as transparent. Eqs. (15) and (16) have one interesting implication, however. If we assume that $\gamma$ is constant, Eq. (16) can still have physically acceptable solutions with finite $\phi$ for all values of $\Omega$, except for only one singular point at $\Omega=1$. These solutions disappear in case $T_{1}=T_{2}=T_{0}$ implying $T_{e}(\xi)=T_{0}$ and consequently $\gamma=1$, where then only a potential $\phi$ independent of $\xi$ is acceptable. For either sign, the factor $\left(\Omega^{2}-1\right)$ will formally act as to re-scale the $\xi$ variation of a solution of Eq. (9) or (11). We have also investigated these cases by the particle in cell simulations, but the results will be discussed in a different context, as they are not directly relevant for the central ideas of this work.

Acknowledgements. This work was in part supported by a grant from the Norwegian Research Council (NFR) and by a Strategic University Programme (SUP).

Topical Editor M. Lester thanks J.E. Wahlund and another referee for their help in evaluating this paper.

\section{References}

Barston, E. M., Electrostatic oscillations in inhomogeneous cold plasmas, Ann. Phys. (NY), 29, 282, 1964.

Birdsall, C. K., and A. B. Langdon, Plasma Physics via Computer Simulation, McGraw-Hill, New York, 1985.

Blackstock, D. T., Propagation of plane sound waves of a finite amplitude in nondissipative fluids, J. Acoust. Soc. Am., 34, 9, 1962.

Børve, S., H. L. Pécseli, and J. Trulsen, Ion phase-space vortices in 2.5 dimensional simulations., J. Plasma Phys., 2000, (in press).

Das, A. C., and J. Fejer, Resonance instability of small-scale fieldaligned irregularities, J. Geophys. Res., 84, 6701, 1979.

Dawson, J. M., Particle simulation of plasmas, Rev. Modern Phys., 55, 403, 1983.

Drago, F. C., O. Engvold, and E. Jensen, The prominence-corona transition region in transverse magnetic fields, Solar Phys., 139, 47, 1991

Dysthe, K. B., E. Mjølhus, H. L. Pécseli, and K. Rypdal, A thermal oscillating two-stream instability; Phys. Fluids, 26, 146, 1983.

Feldman, W. C., J. R. Asbridge, S. J. Bame, and M. D. Montgomery, Double ion streams in the solar wind, J. Geophys. Res., 78, 2017, 1973.

Huld, T., H. L. Pécseli, and J. J. Rasmussen, Ion-acoustic wave propagation in plasma with ion beams having a finite cross section, IEEE Trans. Plasma Phys., 18, 149, 1990.

Kadomtsev, B. B., Plasma turbulence, Academic Press, New York, 1965.

Kadomtsev, B. B., and V. I. Karpman, Nonlinear waves; Sov. Phys. Usp., 14, 40, 1971.

Motley, R. W., Q Machines, Academic Press, New York, 1975.

Nilsson, H., S. Kirwood, and T. Moretto, Incoherent scatter radar observations of the cusp acceleration region and cusp field aligned currents. J. Geophys. Res., 103, 26 721, 1998.

Pécseli, H. L., Solitons and weakly nonlinear waves in plasmas, IEEE Trans. Plasma Sci., PS-13, 53, 1985.

Peñano, J. R., G. J. Morales, and J. E. Maggs, Drift-Alfvén fluctuations associated with a narrow pressure striation, Phys. Plasmas, 7, 144, 2000.

Sato, N., G. Popa, E. Märk, R. Schrittwieser, and E. Mravlag, Test-wave propagation in the presence of a large-amplitude electron plasma wave, Phys. Rev. Lett., 37, 1684-1687, 1976. 
Uberoi, C., Alfvén waves in inhomogeneous magnetic fields, Phys. Fluids, 15, 1673, 1972.

Wahlund, J.-E., A. I. Eriksson, B. Holback, M. H. Boehm, J. Bonell, P. M. Kintner, C. E. Seyler, J. H. Clemmons, L. Eliasson, D. J. Knudsen, P. Norquist, and L. J. Zanetti, Broadband ELF plasma emission during auroral energization 1. Slow ion acoustic waves, J. Geophys. Res., 103, 4343, 1998.

Wahlund, J.-E., P. Louarn, T. Chust, H. de Feraudy, and A. Roux, On ion acoustic turbulence and the nonlinear evolution of kinetic Alfvén waves in aurora, Geophys. Res. Lett., 21, 1831, 1994a.

Wahlund, J.-E., P. Louarn, T. Chust, H. de Feraudy, A. Roux, B. Holback, B. Cabrit, A. I. Eriksson, P. M. Kintner, M. C. Kelley, J. Bonell, and S. Chesney, Observations of ion acoustic fluctuations in the auroral topside ionosphere by the FREJA S/ C, Geophys. Res. Lett., 21, 1835, $1994 \mathrm{~b}$.

Whitham, G. B., Linear and nonlinear waves, John Wiley \& Sons, New York, 1974. 